\title{
AtTi Sociali
}

\section{Dott. Mauro Agosti}

Il 20 gennaio 2017 è deceduto prematuramente a Brescia, per un improvviso malore, il consocio dott. Mauro Agosti; aveva 46 anni. Nato a Brescia l'8 marzo 1970, si era laureato in Scienze agrarie presso l'Università degli Studi di Milano. Come agronomo si occupava di assistenza tecnica ad aziende agricole ed era esperto di specie d'insetti alloctone invasive tra cui la diabrotica del mais e la cimice asiatica. Appassionato di montagna, entomologia e biospeleologia, era anche un apprezzato specialista di Coleotteri Carabidi. Della nostra Società fu membro quasi ininterrottamente per oltre un ventennio, dal 1994 fino alla morte. La sua attività di ricerca, i progetti e la promettente produzione scientifica sono stati purtroppo interrotti dalla precoce scomparsa. Di recente una specie nuova di Coleottero Curculionide, da lui rinvenuta, gli era stata dedicata: Baldorhynchus agostianus Bellò \& Osella, 2016. Al di là dei risultati di ricerca e delle pubblicazioni entomologiche, in coloro che lo hanno conosciuto e hanno goduto della sua amicizia resta il ricordo di una persona aperta, disponibile e leale, di non comuni qualità umane, e il forte rimpianto per averlo perduto troppo presto. Ai famigliari la Società Entomologica porge le condoglianze più sentite.

\section{Dott. Carlo Pesarini}

Carlo Pesarini, nato a Milano il 12 marzo 1946, è deceduto il 29 dicembre 2017 a Ferrara, dove, per i postumi di un ictus che lo aveva colpito nel 2015, si era trasferito per poter essere meglio assistito dal fratello Fausto, a lungo Direttore del Museo di Storia Naturale della città estense.

Carlo si era appassionato all'entomologia fin da ragazzino e nel 1962, appena sedicenne, si iscrisse alla nostra Società, di cui rimase affezionato socio per 55 anni, ricoprendo anche la carica di Consigliere dal 2002 al 2011. Laureatosi in Scienze Naturali nel 1978, si era specializzato ben presto nello studio dei Coleotteri Curculionidi, e in seguito dei Cerambicidi, non perdendo però mai di vista molte altre famiglie di Coleotteri, gli Imenotteri Sinfiti e infine gli Aracnidi, di cui cominciò ad occuparsi quando fu assunto come Conservatore degli Invertebrati nel Museo Civico di Storia Naturale di Milano, carica che ricoperse dal 1982 al 2013.

A fianco di una qualificata ed apprezzata produzione tassonomica Pesarini ci ha lasciato anche un importante lotto di utilissime tabelle dicotomiche per la classificazione di molte famiglie di Coleotteri italiani, stampate sull'Informatore del Giovane Entomologo, edito dalla nostra Società, e arricchite da disegni in bianco e nero; è stato inoltre autore di diversi apprezzati volumetti (alcuni in collaborazione) della serie "Insetti della fauna italiana", con tutte le specie illustrate a colori, pubblicati dalla rivista Natura di Milano. Le tavole sopra citate sono tutte originali e dovute all'abilità grafica e pittorica di Carlo, una delle tante sue capacità professionali.

$\mathrm{Al}$ fratello Fausto, anch'egli nostro socio, ed ai familiari vanno le più sentite condoglianze della Società.

\section{Prof.ssa Rosa Priore}

Nata a Portici il 6 Agosto 1930, Rosa Priore si laureò in Scienze naturali nel 1959, iniziando dal 1962 l'attività di ricercatrice presso l'allora Istituto di Entomologia Agraria di Portici.

Nel corso della sua carriera universitaria, terminata nel 2005, si è interessata di vari aspetti dell'entomologia agraria, e in particolare della morfologia e della biologia di specie fitofaghe, oltre che dei loro parassitoidi e predatori; il suo primo lavoro è stato, infatti, uno studio morfo-biologico sulla Rodolia cardinalis. Dagli anni '70 ha tenuto i corsi di Apicoltura e Bachicoltura e, in seguito, anche uno dei corsi di Entomologia agraria.

Ha pubblicato, tra il 1963 e il 2005, 117 lavori, tra i quali spiccano diversi contributi dedicati alla revisione degli Apoidei conservati nelle collezioni scientifiche dell'ex-Istituto di Entomologia, revisione, realizzata in collaborazione con vari specialisti europei, che le permise di riorganizzare i reperti in una raccolta di una settantina di cassette contenenti ca. 500 specie italiane. È stata Direttore del Dipartimento di Entomologia e Zoologia Agraria nel triennio 1996-1999. 
Con il suo deciso contributo fu realizzata la riqualificazione di ambienti e arredi che ospitano attualmente le importanti collezioni entomologiche conservate nel sito reale di Portici. A seguito dell'istituzione nel 2000 del Museo di Entomologia Filippo Silvestri, divenuta struttura autonoma inserita nel Sistema museale di Ateneo, ne fu il primo Direttore fino al 2005.

E' stata iscritta alla nostra Società dal 1968 al 1991.

Si è spenta a Portici il 17 marzo 2018. Ai familiari le più sentite condoglianze da parte della Società Entomologica Italiana.

\section{Assemblea Generale Ordinaria del 7 aprile 2018}

L'Assemblea Generale Ordinaria della Società Entomologica Italiana ha luogo sabato 7 aprile 2018, alle ore 15, nella sede operativa di Corso Torino 19/4 scala A, Genova, per deliberare sul seguente

\section{ORDINE DEL GIORNO}

1) Convalida dei soci presentati dal Consiglio

2) Elezioni del Consiglio Direttivo

3) Comunicazioni della Presidenza

4) Relazione dei membri del Consiglio

5) Pubblicazioni sociali

6) Bilancio consuntivo esercizio 2017 e previsioni per il 2018

7) Nomina di Soci Onorari

8) IX European Congress of Entomology

9) Varie ed eventuali.

Sono presenti 22 soci senza alcuna delega. A presiedere l'Assemblea viene eletto il Presidente della Società, Prof. Francesco Pennacchio.

1) Sono approvati all'unanimità 21 nuovi soci (19 ordinari e 2 studenti).

2) Per procedere allo scrutinio delle schede elettorali viene nominata una Commissione formata dai soci Drr. Giuliano Lo Pinto, Marco Dellacasa e Alessio Trotta, i quali iniziano le operazioni di spoglio delle schede mentre l'Assemblea prosegue i propri lavori.

3) Il Presidente anticipa il punto 8) dell'Ordine del Giorno informando i presenti sull'organizzazione dell'XI Congresso Europeo di Entomologia che si terrà a Napoli nel prossimo mese di luglio. Al momento gli iscritti sono 850 provenienti da 53 nazioni e i contributi presentati sono circa un migliaio.

Tenendo conto che nel 2019 ricorrerà il $150^{\circ}$ anniversario della fondazione della nostra Società, si propone di indire un'Assemblea straordinaria dei Soci in concomitanza col Congresso Europeo, in modo da celebrare la ricorrenza assieme ai rappresentanti delle altre Società Entomologiche nazionali che interverranno a Napoli, con una breve relazione storica che potrebbe precedere la consegna del Premio Binaghi al vincitore.

4) Il Segretario f.f. comunica che i Soci attualmente sono 460. Il Consigliere Liberti propone ai presenti una riflessione sui motivi che i giovani entomologi possono trovare attualmente per iscriversi alla nostra Società, in un periodo in cui i rapporti interpersonali via web sono diventati così dominanti. Segue un ampio dibattito, da cui emerge l'opportunità di approfondire il problema, cercando magari contatti con il Forum degli Entomologi Italiani, con gli organizzatori della manifestazione di Entomodena, ecc.

5) Il Direttore delle Pubblicazioni riferisce che il fascicolo I del Bollettino è in fase di avanzata preparazione. Si conviene di dare il via alla stampa cartacea dei volumi delle Memorie 94 (2017), già anticipato in versione elettronica lo scorso Dicembre, e 90 (2013), a suo tempo distribuito solo in pdf.

6) Il Bibliotecario, a nome dell'Amministratore-Tesoriere, presenta il bilancio consuntivo 2017 e l'ipotesi di quello preventivo 2018, riportati qui di seguito, che vengono approvati all'unanimità. 


\section{SOCIETA' ENTOMOLOGICA ITALIANA}

\section{BILANCIO CONSUNTIVO 2017}

I. STATO PATRIMONIALE AL 31.12.2017

PATRIMONio Sociale

Immobile Sede Biblioteca Sociale (valore catastale riv.)

Fondo riserva

Mobili \& Attrezzi (pro memoria)

Biblioteca (pro memoria)

LIQUIDITÀ AL 31.12.2017

Conto corrente bancario

Conto corrente postale \begin{tabular}{rr}
$€$ & $405.407,52$ \\
$€$ & $13.000,00$ \\
$€$ & 1,00 \\
$€$ & 1,00 \\
\hline$€$ & $418.409,52$ \\
$€$ & $12.667,75$ \\
$€$ & $12.328,80$
\end{tabular}

$€ \quad 24.996,55$

Totale $€ \quad 443.406,07$

\section{RENDICONTO DI CASSA AL 31.12.2017}

INTROITI

1. Liquidità al 31.12.2016

2. Quote sociali (importi netti)

3. Contributo 5\%o ex-IRPEF

4. Contributo ministeriale

5. Rimborsi

\begin{tabular}{lr}
$€$ & $20.250,43$ \\
$€$ & $17.622,50$ \\
$€$ & $4.278,20$ \\
$€$ & $7.164,65$ \\
$€$ & $2.506,80$ \\
\hline$€$ & $51.822,58$
\end{tabular}

ESBORSI

6. Conto economico

7. Costo pubblicazioni sociali (stampa, confezione \& spedizione)

$€ \quad 12.006,50$

$€ 14.819,53$

LIQUIDITÀ FINALE AL 31.12.2017 (come da stato patrimoniale)

8. Accantonamento a fronte rischi

RATEI PASSIVI AL 31.12.2017

Spese pubblicazione (a calcolo)

Stampa, confezione e spedizione

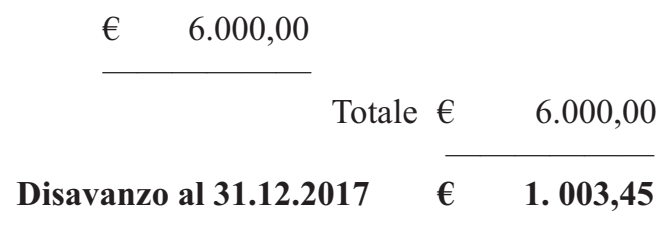


III. DIMOSTRAZIONE DEL CONTO ECONOMICO 2017

6.1 - Gestione ordinaria Sede Sociale

6.2 - Biblioteca Sociale

6.3 - Fondo minute spese Segreteria

6.4 - Fondo minute spese Biblioteca

6.5 - Fondo minute spese Amministrazione

6.6 - Postali e bancarie ordinarie

6.7 - Spese spedizioni

6.8 - Assicurazioni diverse

6.9 - Imposte e tasse

Saldo al 31.12.2017 (come da rendiconto di cassa)
$€ \quad 5.097,99$

$€ \quad 3.117,77$

$€ \quad 200,00$

$€ \quad 500,00$

$€ \quad 300,00$

$€ \quad 653,84$

$€ \quad 728,00$

$€ \quad 625,90$

$€ \quad 783,00$

$€ \quad 12.006,50$

\section{BILANCIO PREVENTIVO ANNO 2018}

INTROITI

1. Quote sociali

2. Utilizzo fondo di riserva

\begin{tabular}{rr}
$€$ & $17.000,00$ \\
$€$ & $20.000,00$ \\
\cline { 2 - 2 } Totale $€$ & $37.000,00$
\end{tabular}

ESBORSI

3. Ratei passivi al 31.12.2017

4. Spese pubblicazione (stampa, confezione, spedizione)

5. Biblioteca sociale

6. Spese generali di gestione, incl. imposte e tasse

\begin{tabular}{rr}
$€$ & $6.000,00$ \\
$€$ & $17.000,00$ \\
$€$ & $6.000,00$ \\
$€$ & $8.000,00$ \\
\cline { 2 - 2 } Totale & 37.000,00
\end{tabular}

L'Amministratore

(Giulio Gardini)
Il Bibliotecario

(Antonio Rey)

7) Il Presidente comunica che nell'ultima riunione del Consiglio Direttivo si era proposto di nominare Soci Onorari il Prof. Augusto Vigna Taglianti e il Rag. Giovanni Dellacasa, per i meriti da loro acquisiti nei confronti della Società durante i lunghi anni in cui furono eletti rispettivamente Presidente ed Amministratore. L'Assemblea approva all'unanimità.

8) Vedasi il punto 2)

9) Nulla da segnalare.

$\mathrm{Al}$ termine dello spoglio delle schede gli scrutatori presentano al Presidente, che ne dà lettura all'Assemblea, i risultati delle elezioni alle cariche sociali per il triennio 2018-2020. 
Per il Consiglio Direttivo.

Schede pervenute 124; schede valide 124. Risultano eletti:

Presidente:

Francesco Pennacchio (voti 113)

Vice Presidente:

Roberto Poggi (116)

Segretario:

Davide Badano (105)

Amministratore/Tesoriere:

Giulio Gardini (111)

Bibliotecario:

Antonio Rey (108)

Direttore delle Pubblicazioni:

Pier Mauro Giachino (111)

Consiglieri:

Alberto Ballerio (88)

Andrea Battisti (91)

Marco A. Bologna (94)

Achille Casale (100)

Marco Dellacasa (79)

Loris Galli (66)

Gianfranco Liberti (71)

Bruno Massa (96)

Massimo Meregalli (75)

Luciana Tavella (77)

Stefano Zoia (78)

Revisori dei Conti:

Enrico Gallo (95)

Sergio Riese (92)

Giuliano Lo Pinto (90)

Revisori dei Conti supplenti:

Giovanni Tognon (88)

Marco Terrile (78)

Si precisa che nell'ordine di preferenze per l'undicesimo Consigliere due candidati (Loris Galli, socio dal 2003, e Morena Casartelli, socia dal 2014) hanno ottenuto l'identico numero di 66 voti; è stato eletto il socio con maggiore anzianità di iscrizione.

Per il Comitato di Coordinamento della Sezione Agraria.

Schede pervenute 38; schede valide 38 (Coordinatore) e 28 (Comitato). Risultano eletti:

Coordinatore:

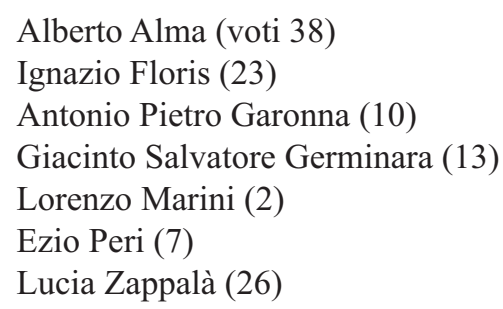

Antonio Pietro Garonna (10)

Giacinto Salvatore Germinara (13)

Lorenzo Marini (2)

Ezio Peri (7)

Lucia Zappalà (26)

Comitato di Coordinamento Ignazio Floris (23)

Si ricorda che il Coordinatore di Sezione è di diritto il $12^{\circ}$ Consigliere della S.E.I.

Si precisa che, mentre per la scelta del Coordinatore non ci sono stati voti dispersi, per quella dei componenti del Comitato ben 10 votanti su 38 hanno espresso 4, 5 o anche 6 preferenze rispetto alle 3 previste e richieste sulla scheda, rendendo di fatto nulle le loro espressioni di voto. Per tale motivo le schede scrutinabili per il Comitato sono state solo 28 .

Esauriti gli argomenti all'ordine del giorno, il Presidente dichiara chiusa l'assemblea alle ore 17,15. 


\section{Nel corso dell'ultima assemblea generale ordinaria sono stati ammessi i seguenti nuovi soci:}

\section{PER IL 2017}

Dr. Giacomo PURROMUTO, Via Ignazio Rosa 30, 97019 Scoglitti (RG) (Entomologia agraria).

\section{PER IL 2018}

Prof. Gianfranco ANFORA, Via Rovereto 6, 38010 Mezzocorona (TN) (Entomologia agraria).

Dr. Leonardo BACCI, Viale Angelo Masini 36, 40126 Bologna (BO) (Lepidoptera, Rhynchota Aphidoidea, Thysanoptera Thripidae).

Dr. Paolo BIELLA, Via Beretta 5, 24050 Calcinate (BG) (Hymenoptera e Diptera impollinatori)

Dr. Arturo COCCO, Via Ugo La Malfa 52, 07100 Sassari (SS) (Entomologia agraria).

Dr. Mario CONTARINI, Via del Collegio 13, 01100 Viterbo (VT).

Dr. Elia GATTO, Via Benaco 22, 30176 Malcontenta (VE) (Cognizione numerica negli Invertebrati)

Dr. Marco GHISOLFI, Via Invalidi del Lavoro 20, 26100 Cremona (CR) (Lepidoptera).

Dr. Costanza JUCKER, Istituto di Entomologia Agraria dell'Università, Via Celoria 2, 20136 Milano (MI) (Controllo specie esotiche; lotta biologica; studio specie per produzione di proteine).

Dr. Isabel MARTINEZ SAÑUDO, Via Giacomo Leopardi 10, 35010 Cadoneghe (PD).

Prof. Ezio PERI, Dipartimento SAAF, Università di Palermo, Viale delle Scienze Ed. 5, 90128 Palermo (PA).

Dr. Riccardo POLONI, Via Palmiro Togliatti 16, 41043 Formigine (MO) (Col.: Cerambycidae, Oedemeridae) (Socio studente).

Dr. Gianandrea SALERNO, Dipartimento di Scienze Agrarie e Ambientali, Borgo XX Giugno, 06121 Perugia (PG).

Dr. Giacomo SANTOIEMMA, Via Finco 25/A, 35010 Campo San Martino (PD) (Ecologia spaziale; Dipt.: Drosophila suzukii; Hem. Aphrophoridae).

Dr. Hannes SCHULER, Via Dreiland 1, 39025 Plaus (BZ) (Simbiosi; ecologia molecolare; protezione delle piante).

Dr. Roberto SINDACO, Via Fatebenefratelli 4, 10137 Torino (TO) (Odonata, Orthoptera, Lepidoptera ital.).

Dr. Paola TIRELLO, Via Paolo Veronese 1/1, 30033 Noale (VE) (Insetti di nuova introduzione; Orthoptera).

Dr. Gregorio VONO, Contrada Chiusa snc, 88069 Staletti (CZ) (Diagnostica molecolare Thysanoptera; biologia e riproduzione Thripidae) (Socio studente).

Dr. Giulia ZANETTIN, Via dell'Albarazzo 10/2, 36060 Schiavon (VI).

Inoltre: Antonino FENIO e Giuseppe PAGANI (Dati anagrafici al momento non comunicati). 